\title{
Macrocycle opening in crown ethers. Synthesis of thiazapodands from 4'-formylbenzothiacrown ethers
}

\author{
Svetlana N. Dmitrieva, Marina V. Churakova, Artem I. Vedernikov, and Sergey P. \\ Gromov* \\ Photochemistry Center of the Russian Academy of Sciences, 7A Novatorov str., Moscow 119421, \\ Russia \\ E-mail:gromov@photonics.ru

\section{Dedicated to Academician Professor Oleg N. Chupakhin on the occasion of his $70^{\text {th }}$ birthday} \\ (received 15 July 04; accepted 01 Oct 04; published on the web 08 Oct 04)
}

\begin{abstract}
A method for the synthesis of thiazapodands from 4'-formylbenzothiacrown ethers was developed. It involves nucleophilic regioselective cleavage of the macrocycle by heating with $\mathrm{MeNH}_{2}$ and $\mathrm{MeNH}_{3}{ }^{+} \mathrm{Cl}^{-}$.
\end{abstract}

Keywords: Macrocycle opening, formylbenzothiacrown ethers, methylamine, podands

\section{Introduction}

Crown compounds are capable of selective binding of metal ions, organic cations, and neutral molecules. This capability underlies the use of crown compounds as selective ligands for metal cations, ${ }^{1}$ including fluorescent and photochromic ones, ${ }^{2}$ for the extraction and separation of metal cations, ${ }^{3}$ for ion transport through membranes, in ion-selective electrodes, ${ }^{4}$ and so on .

Currently, an intensive search for new types of crown compounds which are capable of efficient and selective complexation in various media is in progress. Crown compounds containing combinations of $\mathrm{O}-, \mathrm{N}$-, and $\mathrm{S}$ - atoms within the macrocycle attract steady interest, as they are able to form strong complexes with transition and heavy metal ions. ${ }^{5}$

The main approach to the synthesis of macroheterocyclic compounds is based on the reaction of two acyclic fragments (so-called, " $1+1$ condensation"). Other methods for the construction of macroheterocycles have been much less studied.

We have shown that the formyl- and nitro- derivatives of benzocrown ethers undergo nucleophilic opening of the macrocycle under the action of amines to give open-chain analogs of crown ethers (podands). ${ }^{6}$ The resulting podands were used for the synthesis of benzoazacrown 
ethers whose macrocycle contains a nitrogen atom conjugated with the benzene ring $^{7}$ (Scheme 1).

We are developing a new strategy for the synthesis of functional derivatives of azacrown ethers from the nitrogen-containing podands which are formed upon nucleophilic opening by amines of the macrocycle in the available crown ethers used as synthons. This strategy appears a promising alternative to the existing methods for the synthesis of 1-aza-2,3-benzocrown ethers. ${ }^{8}$

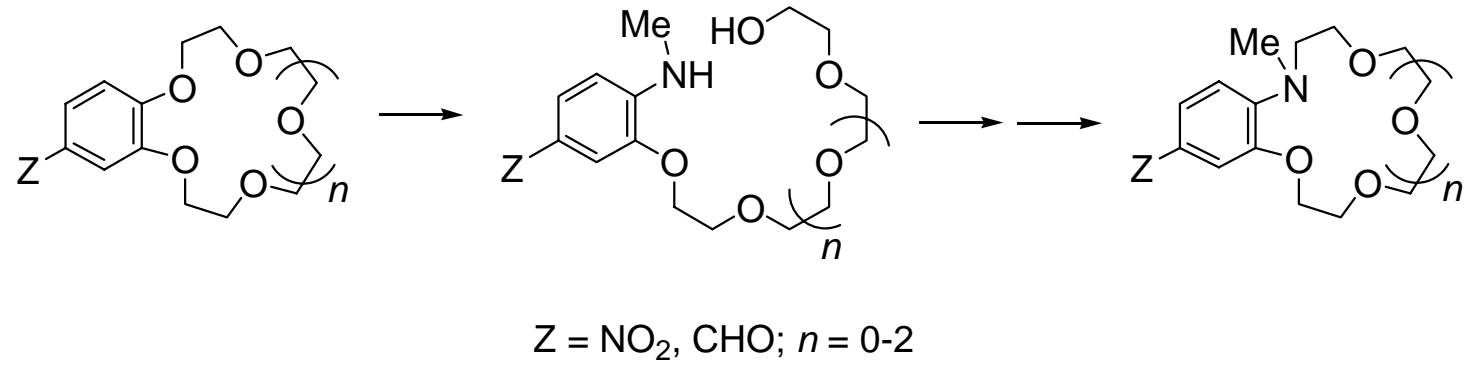

Scheme 1. Synthetic route to $N$-methylbenzoazacrown ethers.

\section{Results and Discussion}

In this work, we have studied the synthesis of a series of thiazapodands from 4'formylbenzothiacrown ethers which have various combinations of $\mathrm{O}$ - and $\mathrm{S}$ - atoms in the macrocycle.

It was found that heating of the formylbenzothiacrown ethers $\mathbf{1 a}-\mathbf{e}$ with an ethanolic solution of $\mathrm{MeNH}_{2}$ and $\mathrm{MeNH}_{3}{ }^{+} \mathrm{Cl}^{-}$followed by hydrolysis of the reaction mixture with dilute acid affords the podands $\mathbf{5 a - e}$ in $39-90 \%$ yields (Scheme 2).

In our opinion, the reaction of methylamine or its hydrochloride with $\mathbf{1}$ initially gives the iminium derivative of the crown-containing benzaldehyde $\mathbf{2}$, which is more activated toward nucleophilic substitution in the para- position than the starting compound. This is apparently followed by the addition of $\mathrm{MeNH}_{2}$ to compound $\mathbf{2}$ to give $\sigma$ - complex $\mathbf{3}$ and elimination of the alkoxy group to give podand $\mathbf{4}$. Treatment of $\mathbf{4}$ with a dilute acid results in hydrolysis of the iminium group, giving rise to the target compounds $\mathbf{5 a - e .}$

It was found that the yields of the resulting thiazapodands 5a-e depend on the size of the macrocycle in the initial benzothiacrown ethers, 1a-e. The highest yields were observed for podands 5b,e prepared from benzothia-15-crown-5 ethers 1b,e. However, we found no substantial differences between the reactivities of compounds $\mathbf{1 b}$ and 1e. Upon a decrease or increase in the size of the benzothiacrown macrocycle in 1a,c,d the yields of the podands 5a,c,d diminish. 
The structures of the resulting compounds were established by ${ }^{1} \mathrm{H}-$ and ${ }^{13} \mathrm{C}-\mathrm{NMR}$, IR spectroscopy, and mass spectrometry, including high-resolution mass spectrometry, and confirmed by elemental analysis data.

\section{Conclusions}

We have developed a method for the synthesis of thiazapodands based on the nucleophilic regioselective opening by methylamine of the macrocycle in readily available formyl derivatives of benzothia- and benzodithiacrown ethers. The resulting podands may prove promising starting compounds for the synthesis of new benzothiazacrown ethers containing a nitrogen atom conjugated with the benzene ring in the macrocycle.

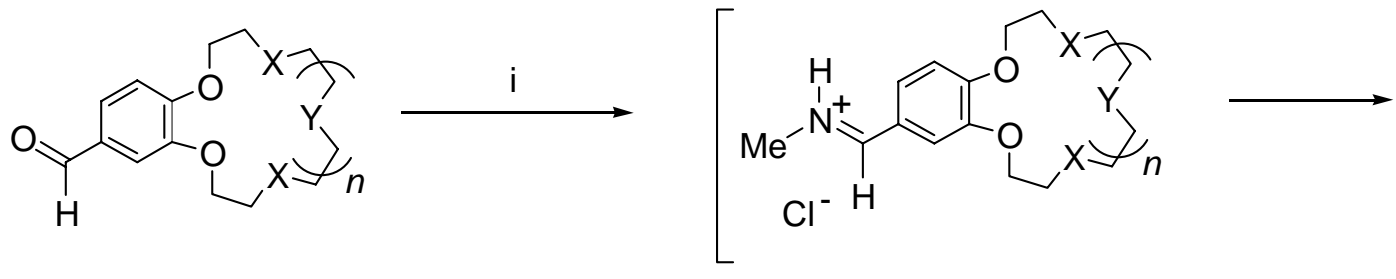

1a-e

2

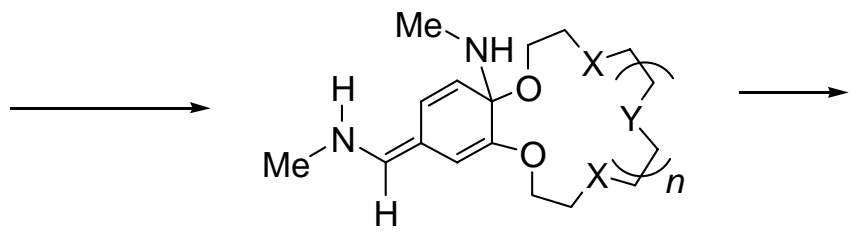

3

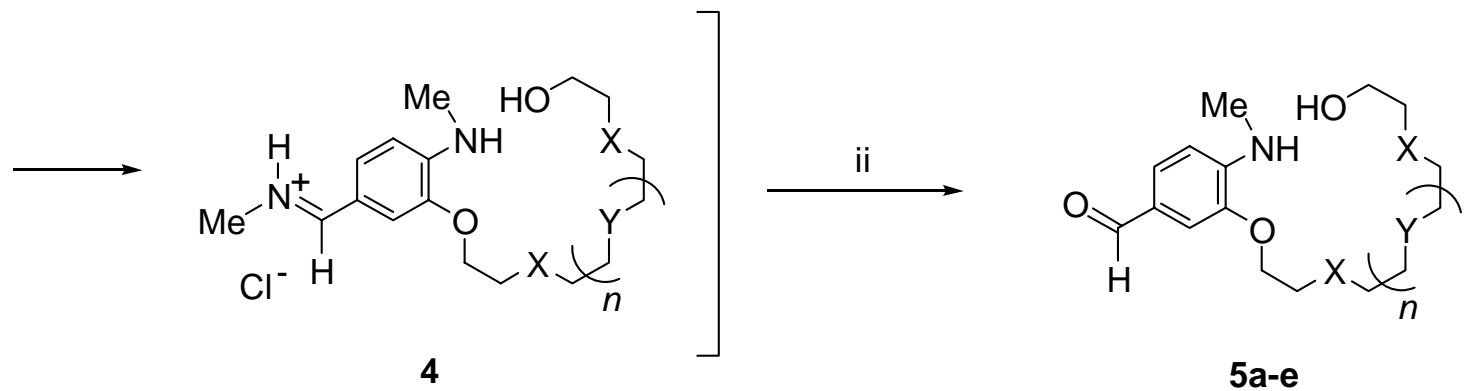

1, 5: $\mathrm{X}=\mathrm{S}, \mathrm{Y}=\mathrm{O} ; n=0$ (a), 1 (b), 2 (c) ), 3 (d)

$\mathrm{X}=\mathrm{O}, \mathrm{Y}=\mathrm{S}, n=1$ (e)

Scheme 2. Reaction conditions: (i) $\mathrm{MeNH}_{3}{ }^{+} \mathrm{Cl}^{-}, \mathrm{MeNH}_{2} / \mathrm{EtOH}$, sealed tube, $160^{\circ} \mathrm{C}, 120 \mathrm{~h}$; (ii) $1.5 \% \mathrm{HBr}, 25^{\circ} \mathrm{C}, 1^{\circ} \mathrm{h}$. 


\section{Experimental Section}

General Procedures. Melting points $\left[{ }^{\circ} \mathrm{C}\right]$ were determined with a MEL-Temp II apparatus in a capillary and were not corrected. 1-D- ${ }^{1} \mathrm{H}$ - and ${ }^{13} \mathrm{C}$ - NMR spectra were recorded on a Bruker DRX500 instrument (500.13 and $125.76 \mathrm{MHz}$, respectively) in acetone- $\mathrm{d}_{6}$ or $\mathrm{CDCl}_{3}$, using the solvent as internal reference $\left(2.05\right.$ and $7.27 \mathrm{ppm}$ for ${ }^{1} \mathrm{H}$, and 29.83 and $77.00 \mathrm{ppm}$ for ${ }^{13} \mathrm{C}$, respectively) at $30^{\circ} \mathrm{C}$; 2D- homonuclear ${ }^{1} \mathrm{H}-{ }^{1} \mathrm{H}$ COSY and heteronuclear ${ }^{1} \mathrm{H}-{ }^{13} \mathrm{C}-\mathrm{COSY}$ (HSQC and $\mathrm{HMBC}$ ) spectra were used to assign the proton and carbon signals. IR spectra were recorded in film on a $\mathrm{KBr}$ glass or in $\mathrm{KBr}$ pellets on Shimadzu IR-470 and Bruker IFS-113V spectrophotometers. Mass spectra were measured on Varian MAT-311A and Finnigan MAT 8430 instruments, and high-resolution mass spectra were recorded on Finnigan MAT-8430 instrument (perfluoroparaffin as a standard) with direct sample inlet into the ionization zone; the energy of ionizing electrons was $70 \mathrm{eV}$. Elemental analyses were performed at the microanalytical laboratory of the A. N. Nesmeyanov Institute of Organoelement Compounds in Moscow, Russia. The course of the reactions was monitored by TLC on Merck DC-Alufolien Kieselgel $60 \mathrm{~F}_{254}$ plates. Column chromatography was performed with Merck Kieselgel 60 (0.063-0.100 mm).

The starting formyl derivatives of benzothia- and benzodithiacrown ethers 1a-e were prepared by known methods. ${ }^{9}$

Synthesis of thiazapodands 5a-e. A mixture of benzocrown ether $1 \mathrm{a}-\mathbf{e}(1 \mathrm{mmol}), \mathrm{MeNH}_{3}{ }^{+} \mathrm{Cl}^{-}$ $(10 \mathrm{mmol})$ and $10 \mathrm{ml}$ of a $35 \%$ solution of $\mathrm{MeNH}_{2}$ in anhydrous $\mathrm{EtOH}$ was heated in a sealed tube for $120 \mathrm{~h}$ at $160^{\circ} \mathrm{C}$ (oil bath). The tube was opened, the mixture was concentrated in vacuo, $35 \mathrm{ml}$ of a $1.5 \%$ aqueous solution of $\mathrm{HBr}$ was added to the dry residue, and the mixture was left for $1 \mathrm{~h}$. A $5 \%$ aqueous solution of $\mathrm{KOH}$ was added to bring the mixture to $\mathrm{pH} 12$, and the reaction products were extracted with EtOAc. The extracts were concentrated in vacuo and the residue purified by column chromatography on $\mathrm{SiO}_{2}$. Compound 5a was eluted with a benzeneEtOAc mixture, 5:1; compounds 5b,e by a benzene-EtOH mixture, 20:1; and compounds 5c,d by EtOAc.

3-[2-(\{2-[(2-Hydroxyethyl)sulfanyl]ethyl\}sulfanyl)ethoxy]-4-(methylamino)benzaldehyde (5a). Yield 39\%, mp 91-93 ${ }^{\circ} \mathrm{C} . \mathrm{R}_{f} 0.46\left(\mathrm{C}_{6} \mathrm{H}_{6}-\mathrm{EtOAc}, 1: 1\right) . \mathrm{IR}(\mathrm{KBr}): v\left[\mathrm{~cm}^{-1}\right] 3494,3335(\mathrm{NH}$, $\mathrm{OH}), 1655(\mathrm{CH}=\mathrm{O}) .{ }^{1} \mathrm{H} \mathrm{NMR}\left(\mathrm{CDCl}_{3}\right): \delta 2.66$ (br. s, $\left.1 \mathrm{H}, \mathrm{OH}\right), 2.74\left(\mathrm{t}, J=6.1 \mathrm{~Hz}, 2 \mathrm{H}, \mathrm{CH}_{2} \mathrm{~S}\right)$, 2.79 (m, $4 \mathrm{H}, 2 \mathrm{CH}_{2} \mathrm{~S}$ ), 2.93 (br. s, $3 \mathrm{H}, \mathrm{MeN}$ ), 2.95 (t, J=6.7 Hz, $2 \mathrm{H}, \mathrm{CH}_{2} \mathrm{~S}$ ), 3.74 (t, $J=6.1$ $\left.\mathrm{Hz}, 2 \mathrm{H}, \mathrm{CH}_{2} \mathrm{O}\right), 4.22$ (t, J=6.7 Hz, $2 \mathrm{H}, \mathrm{CH}_{2} \mathrm{OAr}$ ), 5.10 (br. s, $\left.1 \mathrm{H}, \mathrm{NH}\right), 6.56$ (d, J = 7.9 Hz, 1 H, H-5), 7.24 (d, $J=1.2 \mathrm{~Hz}, 1 \mathrm{H}, \mathrm{H}-2), 7.38$ (dd, $J=7.9, J=1.2 \mathrm{~Hz}, 1 \mathrm{H}, \mathrm{H}-6), 9.65$ (s, $1 \mathrm{H}$, $\mathrm{CH}=\mathrm{O}) .{ }^{13} \mathrm{C} \mathrm{NMR}\left(\mathrm{CDCl}_{3}\right): \delta 29.53(\mathrm{MeN}), 31.20\left(\mathrm{CH}_{2} \mathrm{~S}\right), 32.04\left(\mathrm{CH}_{2} \mathrm{~S}\right), 32.63\left(\mathrm{CH}_{2} \mathrm{~S}\right), 35.19$ $\left(\mathrm{CH}_{2} \mathrm{~S}\right), 60.95\left(\mathrm{CH}_{2} \mathrm{OH}\right), 67.76\left(\mathrm{CH}_{2} \mathrm{OAr}\right), 107.22(\mathrm{C}-5), 107.81(\mathrm{C}-2), 125.33(\mathrm{C}-1), 129.48$ (C-6), 145.11 and 145.34 (C-3, C-4), $190.26(\mathrm{CH}=\mathrm{O}) . \mathrm{MS}, \mathrm{m} / \mathrm{z}, \%: 315[\mathrm{M}]^{+}$(1), 165 (13), 137 (38), 109 (10), 105 (100), 103 (10), 94 (12), 87 (13), 61 (45), 59 (13), 58 (78). Anal. calcd. for $\mathrm{C}_{14} \mathrm{H}_{21} \mathrm{NO}_{3} \mathrm{~S}_{2}$ (315.45): C, 53.30; H, 6.71; N, 4.44. Found: C, 53.22; H, 6.70; N, 4.21\%. 
3-2-[(2-\{2-[(2-Hydroxyethyl)sulfanyl]ethoxyethyl)sulfanyl]ethoxy\}-4-(methylamino)benzaldehyde

(5b). Yield 90\%, yellow oil, $\mathrm{R}_{f} 0.55\left(\mathrm{C}_{6} \mathrm{H}_{6}-\mathrm{EtOAc}, 5: 1\right)$. IR (KBr): $v\left[\mathrm{~cm}^{-1}\right] 3396(\mathrm{NH}, \mathrm{OH}), 1663(\mathrm{CH}=\mathrm{O})$. ${ }^{1} \mathrm{H}$ NMR (acetone- $\mathrm{d}_{6}$ ): $\delta 2.68\left(\mathrm{t}, J=7.1 \mathrm{~Hz}, 2 \mathrm{H}, \mathrm{CH}_{2} \mathrm{~S}\right), 2.71\left(\mathrm{t}, J=6.8 \mathrm{~Hz}, 2 \mathrm{H}, \mathrm{CH}_{2} \mathrm{~S}\right), 2.80(\mathrm{t}, J=$ $6.5 \mathrm{~Hz}, 2 \mathrm{H}, \mathrm{CH}_{2} \mathrm{~S}$ ), 2.87 (br. s, $\left.1 \mathrm{H}, \mathrm{OH}\right), 2.94$ (d, J=5.1 Hz, $3 \mathrm{H}, \mathrm{MeN}$ ), 3.02 (t, J=6.5 Hz, 2 $\mathrm{H}, \mathrm{CH}_{2} \mathrm{~S}$ ), 3.62 (t, $J=6.7 \mathrm{~Hz}, 2 \mathrm{H}, \mathrm{CH}_{2} \mathrm{O}$ ), 3.66 (br. t, $J=6.5 \mathrm{~Hz}, 4 \mathrm{H}, 2 \mathrm{CH}_{2} \mathrm{O}$ ), 4.27 (t, $J=6.6$ $\mathrm{Hz}, 2 \mathrm{H}, \mathrm{CH}_{2} \mathrm{OAr}$ ), 5.70 (br. s, $\left.1 \mathrm{H}, \mathrm{NH}\right), 6.64$ (d, J=8.1 Hz, $\left.1 \mathrm{H}, \mathrm{H}-5\right), 7.27$ (d, J = 1.4 Hz, $1 \mathrm{H}$, $\mathrm{H}-2), 7.43$ (dd, $J=8.1, J=1.4 \mathrm{~Hz}, 1 \mathrm{H}, \mathrm{H}-6), 9.68$ (s, $1 \mathrm{H}, \mathrm{CH}=\mathrm{O}) .{ }^{13} \mathrm{C}-\mathrm{NMR}$ (acetone-d 6 ): $\delta$ $29.55(\mathrm{MeN}), 31.79\left(\mathrm{CH}_{2} \mathrm{~S}\right), 31.99\left(\mathrm{CH}_{2} \mathrm{~S}\right), 32.24\left(\mathrm{CH}_{2} \mathrm{~S}\right), 35.53\left(\mathrm{CH}_{2} \mathrm{~S}\right), 62.25\left(\mathrm{CH}_{2} \mathrm{OH}\right), 69.02$ $\left(\mathrm{CH}_{2} \mathrm{OAr}\right), 71.36\left(\mathrm{CH}_{2} \mathrm{O}\right), 71.52\left(\mathrm{CH}_{2} \mathrm{O}\right), 107.79(\mathrm{C}-5), 108.98(\mathrm{C}-2), 126.20(\mathrm{C}-1), 128.90$ (C-6), 146.13 and 146.23 (C-3, C-4), 189.89 (CH=O). MS, m/z, \%: 359 [M] $]^{+}$(14), 343 (25), 209 (89), 181 (100), 178 (29), 150 (34), 149 (48), 109 (28), 105 (88), 87 (29), 61 (50). HRMS calcd. for $\mathrm{C}_{16} \mathrm{H}_{25} \mathrm{NO}_{4} \mathrm{~S}_{2}[\mathrm{M}]^{+} 359.1225$. Found 359.1199.

3-[(14-Hydroxy-6,9-dioxa-3,12-dithiatetradecyl)oxy]-4-(methylamino)benzaldehyde (5c). Yield 61\%, yellow oil. $\mathrm{R}_{f} 0.55\left(\mathrm{C}_{6} \mathrm{H}_{6}-\right.$ EtOAc, 5:1). IR ( $\left.\mathrm{KBr}\right): v\left[\mathrm{~cm}^{-1}\right] 3388(\mathrm{NH}, \mathrm{OH}), 1663$ $(\mathrm{CH}=\mathrm{O}) .{ }^{1} \mathrm{H}$ NMR (acetone- $\left.\mathrm{d}_{6}\right): \delta 2.68\left(\mathrm{t}, J=6.6 \mathrm{~Hz}, 2 \mathrm{H}, \mathrm{CH}_{2} \mathrm{~S}\right), 2.69(\mathrm{t}, J=6.6 \mathrm{~Hz}, 2 \mathrm{H}$, $\left.\mathrm{CH}_{2} \mathrm{~S}\right), 2.79$ (t, $\left.J=6.5 \mathrm{~Hz}, 2 \mathrm{H}, \mathrm{CH}_{2} \mathrm{~S}\right), 2.93(\mathrm{~d}, J=5.1 \mathrm{~Hz}, 3 \mathrm{H}, \mathrm{MeN}), 3.01$ (t, $J=6.5 \mathrm{~Hz}, 2 \mathrm{H}$, $\mathrm{CH}_{2} \mathrm{CH}_{2} \mathrm{OAr}$ ), $3.57\left(\mathrm{~m}, 4 \mathrm{H}, 2 \mathrm{CH}_{2} \mathrm{O}\right), 3.60$ (t, $\left.J=6.7 \mathrm{~Hz}, 2 \mathrm{H}, \mathrm{CH}_{2} \mathrm{O}\right), 3.66$ (t, J=6.4 Hz, $2 \mathrm{H}$, $\mathrm{CH}_{2} \mathrm{O}$ ), 3.67 (t, $J=6.5 \mathrm{~Hz}, 2 \mathrm{H}, \mathrm{CH}_{2} \mathrm{O}$ ), 3.83 (br. s, $\left.1 \mathrm{H}, \mathrm{OH}\right), 4.26$ (t, J=6.5 Hz, $2 \mathrm{H}, \mathrm{CH}_{2} \mathrm{OAr}$ ), 5.70 (br. q, $1 \mathrm{H}, \mathrm{NH}), 6.62$ (d, $J=8.2 \mathrm{~Hz}, 1 \mathrm{H}, \mathrm{H}-5$ ), 7.27 (d, $J=1.4 \mathrm{~Hz}, 1 \mathrm{H}, \mathrm{H}-2), 7.43$ (dd, $J=$ $8.2, J=1.4 \mathrm{~Hz}, 1 \mathrm{H}, \mathrm{H}-6), 9.68(\mathrm{~s}, 1 \mathrm{H}, \mathrm{CH}=\mathrm{O}) .{ }^{13} \mathrm{C} \mathrm{NMR}\left(\right.$ acetone-d $\left._{6}\right): \delta 29.76(\mathrm{MeN}), 31.92$ $\left(\mathrm{CH}_{2} \mathrm{~S}\right), 32.13\left(\mathrm{CH}_{2} \mathrm{~S}\right), 32.38\left(\mathrm{CH}_{2} \mathrm{~S}\right), 35.70\left(\mathrm{CH}_{2} \mathrm{~S}\right), 62.37\left(\mathrm{CH}_{2} \mathrm{OH}\right), 69.18\left(\mathrm{CH}_{2} \mathrm{OAr}\right), 70.81$ $\left(\mathrm{CH}_{2} \mathrm{O}\right), 70.87\left(\mathrm{CH}_{2} \mathrm{O}\right), 71.80\left(\mathrm{CH}_{2} \mathrm{O}\right), 72.01\left(\mathrm{CH}_{2} \mathrm{O}\right), 107.94(\mathrm{C}-5), 109.11(\mathrm{C}-2), 126.27(\mathrm{C}-1)$, 129.12 (C-6), 146.25 and 146.35 (C-3, C-4), $190.13(\mathrm{CH}=\mathrm{O}) . \mathrm{MS}, \mathrm{m} / \mathrm{z}, \%: 403[\mathrm{M}]^{+}(17), 372$ (12), 255 (11), 254 (13), 253 (100), 225 (63), 149 (24), 105 (36), 87 (16), 61 (16). HRMS calcd. for $\mathrm{C}_{18} \mathrm{H}_{29} \mathrm{NO}_{5} \mathrm{~S}_{2}[\mathrm{M}]^{+}$403.1487. Found 403.1497.

3-[(17-Hydroxy-6,9,12-trioxa-3,15-dithiaheptadecyl)oxy]-4-(methylamino)benzaldehyde (5d). Yield 49\%, yellow oil. $\mathrm{R}_{f} 0.30$ (EtOAc). IR (KBr): $v\left[\mathrm{~cm}^{-1}\right] 3391(\mathrm{NH}, \mathrm{OH}), 1668(\mathrm{CH}=\mathrm{O})$. ${ }^{1} \mathrm{H}$ NMR (acetone- $\mathrm{d}_{6}$ ): $\delta 2.69\left(\mathrm{~m}, 4 \mathrm{H}, 2 \mathrm{CH}_{2} \mathrm{~S}\right), 2.80\left(\mathrm{t}, J=6.5 \mathrm{~Hz}, 2 \mathrm{H}, \mathrm{CH}_{2} \mathrm{~S}\right), 2.93(\mathrm{~d}, J=5.1$ $\mathrm{Hz}, 3 \mathrm{H}, \mathrm{MeN}), 3.02\left(\mathrm{t}, J=6.5 \mathrm{~Hz}, 2 \mathrm{H}, \mathrm{CH}_{2} \mathrm{~S}\right), 3.56\left(\mathrm{~m}, 4 \mathrm{H}, 2 \mathrm{CH}_{2} \mathrm{O}\right), 3.58\left(\mathrm{~s}, 4 \mathrm{H}, 2 \mathrm{CH}_{2} \mathrm{O}\right)$, $3.60\left(\mathrm{t}, J=6.8 \mathrm{~Hz}, 2 \mathrm{H}, \mathrm{CH}_{2} \mathrm{O}\right), 3.67\left(\mathrm{t}, J=6.5 \mathrm{~Hz}, 4 \mathrm{H}, 2 \mathrm{CH}_{2} \mathrm{O}\right), 4.27$ (t, $J=6.5 \mathrm{~Hz}, 2 \mathrm{H}$, $\mathrm{CH}_{2} \mathrm{OAr}$ ), 5.71 (br. q, $1 \mathrm{H}, \mathrm{NH}$ ), 6.63 (d, J=8.1 Hz, $1 \mathrm{H}, \mathrm{H}-5$ ), 7.27 (br. s, $1 \mathrm{H}, \mathrm{H}-2$ ), 7.43 (br. d, $J=8.1 \mathrm{~Hz}, 1 \mathrm{H}, \mathrm{H}-6), 9.68(\mathrm{~s}, 1 \mathrm{H}, \mathrm{CH}=\mathrm{O}) .{ }^{13} \mathrm{C}$ NMR (acetone-d $\left.{ }_{6}\right): \delta 29.94(\mathrm{MeN}), 32.12$ $\left(\mathrm{CH}_{2} \mathrm{~S}\right), 32.34\left(\mathrm{CH}_{2} \mathrm{~S}\right), 32.59\left(\mathrm{CH}_{2} \mathrm{~S}\right), 35.89\left(\mathrm{CH}_{2} \mathrm{~S}\right), 62.60\left(\mathrm{CH}_{2} \mathrm{OH}\right), 69.45\left(\mathrm{CH}_{2} \mathrm{OAr}\right), 71.05$ $\left(\mathrm{CH}_{2} \mathrm{O}\right), 71.12\left(\mathrm{CH}_{2} \mathrm{O}\right), 71.34\left(2 \mathrm{CH}_{2} \mathrm{O}\right), 72.06\left(\mathrm{CH}_{2} \mathrm{O}\right), 72.26\left(\mathrm{CH}_{2} \mathrm{O}\right), 108.15(\mathrm{C}-5), 109.37$ (C-2), 126.53 (C-1), 129.26 (C-6), 146.50 and 146.59 (C-3, C-4), 190.29 (CH=O). MS, m/z, \%: $447\left[_{\mathrm{M}}^{+}\right]^{+}$(13), 297 (100), 269 (36), 193 (25), 150 (31), 105 (87), 94 (14), 89 (16), 87 (21), 61 (38), 60 (18). HRMS calcd. for $\mathrm{C}_{20} \mathrm{H}_{33} \mathrm{NO}_{6} \mathrm{~S}_{2}[\mathrm{M}]^{+} 447.1749$. Found 447.1753.

3-[2-(2-\{[2-(2-Hydroxyethoxy)ethyl]sulfanyl\}ethoxy)ethoxy]-4-(methylamino)benzaldehyde (5e). Yield 76\%, yellow oil. $\mathrm{R}_{f} 0.41\left(\mathrm{C}_{6} \mathrm{H}_{6}-\right.$ EtOAc, 5:1). IR $(\mathrm{KBr}): v\left[\mathrm{~cm}^{-1}\right] 3373(\mathrm{NH}, \mathrm{OH})$; $1670(\mathrm{CH}=\mathrm{O}) .{ }^{1} \mathrm{H}$ NMR (acetone- $\left.\mathrm{d}_{6}\right): \delta 2.74\left(\mathrm{t}, J=6.7 \mathrm{~Hz}, 2 \mathrm{H}, \mathrm{CH}_{2} \mathrm{~S}\right), 2.77(\mathrm{t}, J=6.6 \mathrm{~Hz}, 2 \mathrm{H}$, 
$\mathrm{CH}_{2} \mathrm{~S}$ ), 2.85 (br. s, $\left.1 \mathrm{H}, \mathrm{OH}\right), 2.93(\mathrm{~d}, J=5.1 \mathrm{~Hz}, 3 \mathrm{H}, \mathrm{MeN}), 3.51$ (m, $\left.2 \mathrm{H}, \mathrm{CH}_{2} \mathrm{O}\right), 3.63$ (m, $4 \mathrm{H}$, $\left.2 \mathrm{CH}_{2} \mathrm{O}\right), 3.71\left(\mathrm{t}, J=6.6 \mathrm{~Hz}, 2 \mathrm{H}, \mathrm{CH}_{2} \mathrm{O}\right), 3.85\left(\mathrm{~m}, 2 \mathrm{H}, \mathrm{CH}_{2} \mathrm{CH}_{2} \mathrm{OAr}\right), 4.22$ (m, $2 \mathrm{H}, \mathrm{CH}_{2} \mathrm{OAr}$ ), 5.74 (br. s, $1 \mathrm{H}, \mathrm{NH}), 6.64$ (d, $J 8.1 \mathrm{~Hz}, 1 \mathrm{H}, \mathrm{H}-5), 7.28$ (d, J=1.4 Hz, $1 \mathrm{H}, \mathrm{H}-2), 7.43$ (dd, $J=$ $8.1, J=1.4 \mathrm{~Hz}, 1 \mathrm{H}, \mathrm{H}-6), 9.68(\mathrm{~s}, 1 \mathrm{H}, \mathrm{CH}=\mathrm{O}) .{ }^{13} \mathrm{C}$ - NMR (acetone-d $\left.\mathrm{d}_{6}\right): \delta 29.77(\mathrm{MeN}), 32.52$ $\left(2 \mathrm{CH}_{2} \mathrm{~S}\right), 62.02\left(\mathrm{CH}_{2} \mathrm{OH}\right), 69.15\left(\mathrm{CH}_{2} \mathrm{OAr}\right), 69.85\left(\mathrm{CH}_{2} \mathrm{O}\right), 71.90\left(2 \mathrm{CH}_{2} \mathrm{O}\right), 73.23\left(\mathrm{CH}_{2} \mathrm{O}\right)$, 108.02 (C-5), 109.60 (C-2), 126.46 (C-1), 129.16 (C-6), 146.64 and 146.68 (C-3, C-4), 190.09 $(\mathrm{CH}=\mathrm{O}) . \mathrm{MS}, \mathrm{m} / \mathrm{z}, \%: 343[\mathrm{M}]^{+}$(100), 195 (64), 194 (44), 193 (73), 151 (63), 150 (71), 149 (59), 148 (41), 87 (49), 61 (45). HRMS calcd. for $\mathrm{C}_{16} \mathrm{H}_{25} \mathrm{NO}_{5} \mathrm{~S},[\mathrm{M}]^{+}$343.1453. Found 343.1455.

\section{Acknowledgments}

This work was supported by the Russian Foundation for Basic Research (project no. 03-0332177), the Presidium of the Russian Academy of Sciences, the Russian Science Support Foundation, and the INTAS (grant no. 2001-0267).

\section{References}

1. (a) Cation Binding by Macrocycles; Inoue, Y.; Gokel, G. W., Eds.; Marcel Dekker: New York, 1990. (b) Izatt, R. M.; Pawlak, K.; Bradshaw, J. S.; Bruening R. L. Chem. Rev. 1991, 91, 1721.

2. (a) de Silva, A. P.; Gunaratne, H. Q. N.; Gunnlaugsson, T.; Huxley, A. J. M.; McCoy, C. P.; Rademacher, J. T.; Rice, T. E. Chem. Rev. 1997, 97, 1515. (b) Alfimov, M. V.; Gromov, S. P. In Applied Fluorescence in Chemistry, Biology, and Medicine; Rettig, W.; Strehmel, B.; Schrader, S.; Seifert, H., Eds., Springer-Verlag: Berlin, 1999; p 161. (c) Valeur, B.; Leray, I. Coord. Chem. Rev. 2000, 205, 3. (d) Shinkai, S. In Comprehensive Supramolecular Chemistry; Gokel, G. W., Ed.; Pergamon: Oxford, 1996; Vol. 1, p 671. (e) Gromov, S. P.; Alfimov, M. V. Izv. Akad. Nauk, Ser. Khim. 1997, 641; Russ. Chem. Bull. 1997, 46, 611 (Engl. Transl.). (f) Mishra, A.; Behera, R. K.; Behera, P. K.; Mishra, B. K.; Behera, G. B. Chem. Rev. 2000, 100, 1973.

3. (a) Yordanov, A. T.; Roundhill, D. M. Coord. Chem. Rev. 1998, 170, 93. (b) Gloe, K.; Graubaum H.; Wust, M.; Rambusch, T.; Seichter, W. Coord. Chem. Rev. 2001, 222, 103.

4. Buhlmann, P.; Pretsch, E.; Bakker, E. Chem. Rev. 1998, 98, 1593.

5. Bradshaw, J. S.; Krakowiak, K. E.; Izatt, R. M. Aza-Crown Macrocycles; John Wiley \& Sons: New York, 1993.

6. (a) Gromov, S. P.; Vedernikov, A. I.; Fedorova, O. A. Izv. Akad. Nauk, Ser. Khim. 1995, 950 [Russ. Chem. Bull. 1995, 44, 923 (Engl. Transl.)]. (b) Gromov, S. P.; Vedernikov, A. I.; Fedorova, O. A. Izv. Akad. Nauk, Ser. Khim. 1996, 687; Russ. Chem. Bull. 1996, 45, 648 (Engl. Transl.). (c) Gromov, S. P.; Dmitrieva, S. N.; Krasnovsky, V. E. Izv. Akad. Nauk, Ser. 
Khim. 1997, 540; Russ. Chem. Bull. 1997, 46, 519 (Engl. Transl.). (d) Gromov, S. P.; Dmitrieva, S. N. Izv. Akad. Nauk, Ser. Khim. 1999, 542; Russ. Chem. Bull. 1999, 48, 537 (Engl. Transl.).

7. (a) Gromov, S. P.; Vedernikov, A. I.; Dmitrieva, S. N. RF Patent 2,161,153, 2000; Chem. Abstr. 2002, 136, 401,792. (b) Gromov, S. P.; Dmitrieva, S. N.; Churakova, M. V. Synthesis 2003, 593. (c) Gromov, S. P.; Dmitrieva, S. N.; Vedernikov, A. I.; Kuz'mina, L. G.; Churakov, A. V.; Strelenko, Yu. A.; Howard, J. A. K. Eur. J. Org. Chem. 2003, 3189. (d) Gromov, S. P.; Dmitrieva, S. N.; Churakova, M. V. RF Patent 2,215,738, 2003.

8. Gromov, S. P.; Dmitrieva, S. N.; Vedernikov, A. I.; Churakova, M. V. Izv. Akad. Nauk, Ser. Khim. 2004, 1362.

9. (a) Fedorova, O. A.; Vedernikov, A. I.; Yescheulova, O. V.; Pershina, Y. V.; Tsapenko, P. V.; Gromov, S. P. Synth. Commun. 2002, 32, 1909. (b) Yescheulova, O. V. Ph.D. Thesis, Moscow, 2001, p 133. 